MULTIPLE SCLEROSIS

\section{Oligodendrocyte studies reveal limits on remyelination}

Previously unseen oligodendrocyte dynamics and heterogeneity could limit remyelination in multiple sclerosis (MS), according to two new studies published in Nature. The findings could provide new therapeutic opportunities.

Repair of myelin in demyelinating disease is thought to involve the generation of new oligodendrocytes. However, this view is based on animal models, and previous work by Jonas Frisén and colleagues has shown that although oligodendrocyte generation occurs in adult rodents, it is limited in healthy adult humans.

"Remyelination is efficient and solely carried out by newly generated oligodendrocytes in rodent models of demyelination," explains Frisén. "We wondered whether oligodendrocyte generation is induced in MS, and whether remyelination is carried out by new oligodendrocytes also in humans."

Using a technique based on ${ }^{14} \mathrm{C}$ incorporation into oligodendrocyte

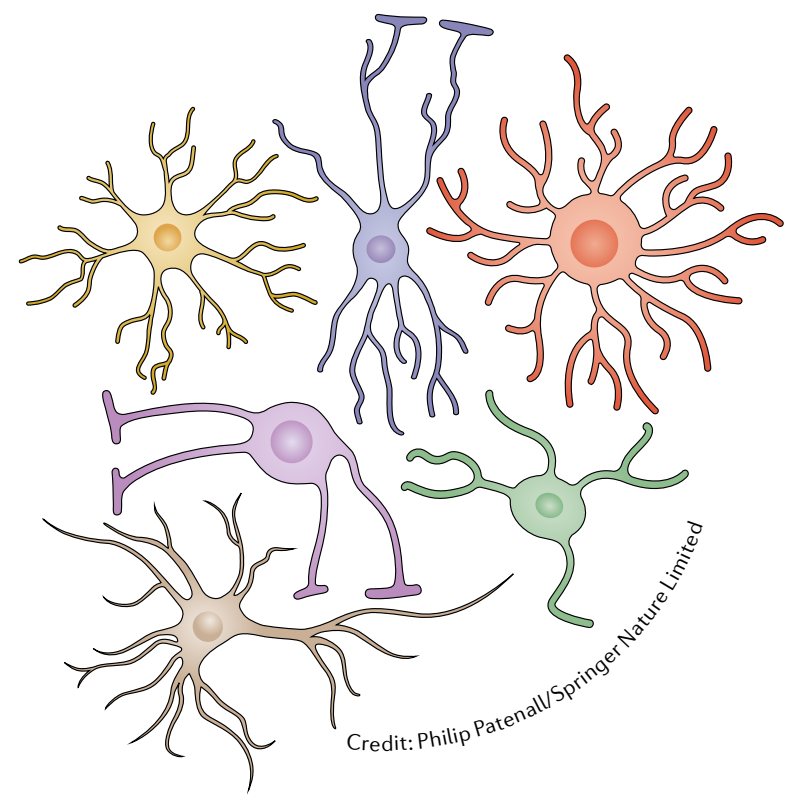

DNA, Frisén and colleagues assessed the ages of oligodendrocytes in post-mortem brain samples from people with MS. No new oligodendrocytes were detected in shadow plaques (lesions in which remyelination is thought to have occurred), an observation that is consistent with the limited oligodendrocyte generation that was previously observed. This finding raises the possibility that old oligodendrocytes remyelinate axons.

"What we found in patients with MS is in stark contrast to the prevailing view based on animal models that all remyelinating oligodendrocytes in shadow plaques are newly made," says Frisén. "We conclude that there is minimal, if any, oligodendrocyte generation in shadow plaques in MS."

The lack of oligodendrocyte generation could explain why early treatment is important in MS, as early intervention would preserve oligodendrocytes. Frisén also thinks the findings point to a therapeutic opportunity. "The lack of generation of new oligodendrocytes poses the question of how this process is blocked," he says. "Identifying the molecular nature of this block may allow the development of therapies that promote oligodendrocyte generation and more efficient remyelination."

The second study built on earlier findings on the molecular characteristics of oligodendrocytes that are present in MS. Previously, using single-cell RNA sequencing (RNA-seq), Gonçalo Castelo-Branco and colleagues had shown that oligodendrocytes in mice were transcriptionally heterogeneous, and that the microglia populations in a mouse model of MS differed from those in wild-type mice.

"We identified unique cell states among oligodendrocyte lineages in the disease models, including oligodendrocyte progenitor cells and mature oligodendrocytes with some characteristics of immune cells," explains Castelo-Branco. "This work led us to investigate whether this was also the case in patients with MS."

Led by Castelo-Branco, Anna Williams and Charles ffrench-Constant, the researchers used single-nucleus RNA-seq to analyse post-mortem white matter from four people with progressive MS and five people without neurological disease. They found that human oligodendrocytes were also transcriptionally heterogenous, and that some oligodendrocyte populations were reduced and some were increased in people with progressive MS.

"The heterogeneity was altered in lesions but also in the normal-appearing white matter, suggesting that the disease is not circumscribed to lesions, but has a wider effect in the CNS," says Williams.

People with MS had a lower number of oligodendrocyte progenitor cells, a difference that could limit remyelination in progressive MS. "This result could also be consistent with the findings from Frisén's group that 'old' oligodendrocytes might contribute to remyelination in shadow plaques," says Castelo-Branco.

The findings also suggest that variations in oligodendrocyte heterogeneity contribute to disease. "If it turns out that heterogeneous oligodendrocytes have distinct functional roles, we may need to consider therapeutic strategies to correct this variation," concludes ffrench-Constant.

Ian Fyfe

ORIGINAL ARTICLES Yeung, M. S. et al. Dynamics of oligodendrocyte generation in multiple sclerosis. Nature https://doi.org/10.1038/s41586-018-0842-3 (2019) | Jakel, S. et al. Altered human oligodendrocyte heterogeneity in multiple sclerosis. Nature https://doi.org/10.1038/s41586-019-0903-2 (2019) 